\title{
Interference Management in Underlay Spectrum Sharing Using Indirect Power Control Signalling
}

\author{
Mohammad G. Khoshkholgh, Keivan Navaie, Senior Member, IEEE, \\ and Halim Yanikomeroglu, Senior Member, IEEE
}

\begin{abstract}
In this paper, we propose an interference management method for underlay spectrum sharing and evaluate its performance. In this method the secondary service has been facilitated by granting passive access to the power control signalling transmitted by the primary network base station. To exploit both slow shadowing and fast fading, the proposed method performs secondary service power management in two phases, each in different time-scales: rate optimal power allocation phase and interference reduction power adjustment phase. In the longer time-scale, rate optimal power allocation phase adaptively allocates the secondary transmit power exploiting the medium-scale channel variations (shadowing effect) of the secondary channel to maximize its capacity. In the shorter timescale, interference reduction power adjustment phase exploits the power control commands transmitted in the primary network to adaptively adjust secondary service transmission power for reducing the effects of the secondary service transmission on the Quality-of-Service (QoS) of the primary service network. The main advantage of this method which is referred to as Adaptive Multiple Time-Scale Power Allocation (AMTPA) is that it does not require direct signaling between the two systems. We further present AMTPA analytical performance evaluation results. Practical considerations are also presented regarding the primary network requirements and its power control feasibility after adopting AMTPA in the secondary network. Extensive simulation results indicate significant improvement in the system performance by using AMTPA. Using simulations we also show how one can set AMTPA parameters so that a certain level of QoS in the primary network is satisfied.
\end{abstract}

Index Terms-Collision probability, DS-CDMA, interference management, underlay spectrum access, spectrum sharing.

\section{INTRODUCTION}

$\mathbf{I}$ $\mathrm{N}$ spectrum sharing a secondary service is able to access the white spaces in a frequency band which is formally allocated to the primary service [1]. White spaces are those parts of the spectrum allocated to the primary user which are under-utilized in some particular times and specific locations. Various schemes are proposed in the literature for spectrum sharing (see e.g., [2] and references therein). Our focus is on the underlay spectrum sharing [3]. In underlay spectrum sharing, the secondary service can always access to the spectrum subject to an interference threshold constraints at the primary service receiver [2], [3].

Manuscript received August 1, 2012; revised November 21, 2012; accepted April 22, 2013. The associate editor coordinating the review of this paper and approving it for publication was L. Deneire.

M. G. Khoshkholgh is with Simula Research Laboratory, Oslo, Norway.

K. Navaie is with the School of Electronic and Electrical Engineering, University of Leeds, Leeds, UK LS2 9JT (e-mail: keivan.navaie@ieee.org).

H. Yanikomeroglu is with the Department of Systems and Computer Engineering, Carleton University, Ottawa, ON, Canada.

Digital Object Identifier 10.1109/TWC.2013.052813121110
To satisfy the interference threshold constraint, in underlay spectrum sharing, Channel State Information (CSI) between the secondary transmitter and receiver (secondary-channel), and between the primary receiver and the secondary transmitter (cross-channel) are required (see, e.g., [4], [5], [6]).

In practice, the cross-channel CSI may be fedback directly by the primary service to the secondary transmitter, measured by the secondary transmitter, or indirectly through a higher layer band manager which coordinate spectrum access [1]. However, the aforementioned scenarios are difficult to be implemented since the primary network has been already deployed and not been designed to provide direct signalling to other networks. To tackle this, different methods have been presented in the related literature.

In [7], [8] a low bit rate signaling channel is considered between the primary and the secondary systems for communicating the channel side information. For measuring the channel gains, pilot signals are transmitted by both systems. However, the pilot transmission and its corresponding signaling channel impose a system overhead that may not be acceptable to the primary network. To resolve this, in [9] a scheme is proposed by which the secondary service estimates the power spectrum of the interference using a multi-taper method. Utilizing a large number of sensors is also proposed in [10] to sniff the radio frequency environment. Such method encounters practical concerns in a civilian wireless network.

Recently approaches based on exploiting the channel feedback in the primary network by the secondary service, have been proposed in the related literature. In such approaches, the feedback is utilized by the secondary network to manage the impact of spectrum sharing on the primary service as well as to enhance the secondary service performance. In [11], a spectrum sharing scenario has been proposed in which the secondary service overhears and exploits the Automatic Repeat Request (ARQ)s signaling in the primary service and accordingly adjusts its access to the spectrum. It is also shown in [11] that this passive overhearing guarantees primary service target rate. Similarly in [12], a feedback-based access scheme is suggested in which the secondary service is allowed to overhear the primary service ARQ channel. If ACK is observed, the secondary service will then access the channel; otherwise access is deferred to reduce almost certain collision outcome between the secondary and primary transmissions. Only one primary transceiver and secondary transceiver is considered and the analysis is conducted for an erasure channel in which primary channels erase probability is changed due to secondary service activity.

Furthermore, in [13] for a system model including a pair 
of secondary and primary services in which the secondary service has access to the quantized channel state information of the primary channel, i.e., the channel between the primary transmitter and receiver, they then formulate an optimization problem to maximize the secondary service capacity subject to primary service lose rate constraint. Having access to this information the secondary service is then able to speculate the rate reduction at the primary service due to its transmission.

In [14] a method has been proposed in which the secondary service is provided with passive access to one information bit that indicates the status of the outage constraint at the primary receiver. However, only one primary transceiver is considered and impact of shadowing is also ignored.

To tackle the cross-channel CSI issue, here we propose an indirect power control signalling method namely Adaptive Multiple Time-Scale Power Allocation (AMTPA) for underlay spectrum sharing over uplink of Direct Sequence Code Division Multiple Access (DS-CDMA) primary networks. In AMTPA power control signalling in the primary system is utilized by the secondary service to adjust its transmit power based on the primary network actual status; thus to manage the interference. Orthogonal frequency division multiple access (OFDM) is utilized by the secondary service to access the available spectrum.

\section{A. DS-CDMA/OFDM Spectrum Sharing}

DS-CDMA is the dominant air interface technique $3 \mathrm{G}$ mobile communications and some Wireless Local Area Network (WLAN) technologies. Therefore, the spectrum sharing over existing DS-CDMA-based networks is anticipated to be one of the spectrum sharing applications in the near future.

From the primary service perspective, the spreading characteristic of DS-CDMA makes it more robust to the narrowband interference created by OFDM secondary users. From the secondary service perspective, OFDM provides the required flexibility to the secondary service to access separate appropriate portions of the spectrum. Since the wireless channel is frequency selective, appropriate portions (i.e., subchannels) might be found for secondary transmission which impose minimum interference on the primary service, and at the same time achieve as much capacity as possible in the secondary system. For instance consider the case that there are some sub-channels with a deep fade over the link between the secondary transmitter and the primary receiver, and a good channel condition over the link between the secondary transmitter and receiver. Such sub-channels, if selected for secondary transmission, result in a high secondary service capacity, and at the same time impose low narrow-band interference over the primary network. The impact of this imposed narrow-band interference is further decreased in the primary network because of DS-CDMA technology. Therefore, DS-CDMA/OFDM combination provides a new degree of freedom by enabling the secondary service to adaptively select appropriate sub-channels.

\section{B. The Proposed Approach}

To utilize AMTPA in an existing network, the only requirement is an indirect inter-system cooperation between the primary network and secondary service. Such indirect intersystem cooperation, in its simplest form, facilitates secondary service access to the corresponding power control commands of a particular user of the primary service which we refer to as the 'probe user'. In fact, the main advantage of the AMTPA is that the primary system does not require to be modified to support AMPTA. However, the scheme does require a degree of cooperation and co-design to enable the secondary users to have access to the corresponding signaling channels to receive the power control commands in the primary system. Comparing to establishing a new direct signaling channel between the primary and secondary systems, AMTPA requires much lower level of cooperation between the two system.

Our focus is spectrum sharing in the uplink of the primary network. That is because most of the modern data applications are asymmetric, i.e., the amount of downlink communications is much higher than that of the uplink. Therefore, for spectrum sharing over $2 \mathrm{G}$ and $3 \mathrm{G}$ systems, the uplink spectrum is most likely under-utilized and can be used for spectrum sharing.

To maintain a certain level of wireless link quality, in DSCDMA systems a power control mechanism is adopted [15]. The transmitter then adjusts its transmit power based on the received power control commands to maintain the link quality. The base station (BS) of the primary service routinely reacts to degrading the wireless link quality by sending suitable power control commands to the affected primary service users. In case of underlay spectrum sharing, the interference caused by the secondary users, if not the only reason, is one of the crucial factors in degrading the link quality in the primary network. Therefore, the power control commands contain information reflecting the interference level in the primary network.

Through listening to power control commands in AMTPA, the secondary service users can adjust their transmit powers to satisfy the link quality constrain in the primary network. The secondary service employs underlay spectrum sharing for accessing a portion of the uplink spectrum, namely $s u b$ channel. A two level time hierarchy consisting of "frames" and "slots" is considered in which, each frame is divided into a number of slots. Secondary service transmission is managed in two phases: rate optimal power allocation phase and interference reduction power adjustment phase, performed in the frame and slot time-scales, respectively.

The rate optimal power allocation phase adaptively allocates the optimal transmit power using the instantaneous status of the secondary channel with the objective of maximizing the capacity of the secondary service without considering the impact of secondary transmission on the link quality in the primary network. Utilizing the power control commands of the primary network, the allocated power is then adjusted in the interference reduction power adjustment phase, so that the required link quality in the primary network is satisfied.

Power adjustment is conducted through increasing/decreasing transmission power with fixed step size $\Delta_{s}$ Watts. Modified versions of AMTPA are also presented to further improve the system performance. We also propose a scheme for selecting the probe user. Interestingly, we show that the probe user can be selected by the primary service based-station based on the channel power gains among the BS and the primary users, and the eigenvalues of the primary users channel matrix. 
We also discuss practical considerations and the feasibility of the power control in the primary network when AMTPA is adopted by the secondary network. This paper provides an instance of the approaches in which the inherent structure of the primary network can be exploited by the secondary service. This is significantly important since using such approaches minimum modifications in the primary network is required to facilitate spectrum sharing.

Simulations are performed to study different performance aspects of the proposed method. To the authors' best knowledge, there is no other fundamentally similar approach to AMTPA; therefore, in our simulations, among other things, we compare the performance of AMTPA with the existing performance bounds in the related literature. The simulation results indicate improvement in the system collision performance by using AMTPA. Using simulations we also show how one can set the AMTPA system parameters so that a certain level of quality-of-service (QoS), e.g., collision probability in the primary network is satisfied.

\section{Contributions}

Our main objective in this paper is to show how the secondary service can exploit one bit power control command at the primary service to guarantee the primary service qualityof-service in terms of outage probability and power control feasibility. The contributions of this paper are as follows:

- A new secondary service power allocation is proposed in which an indirect signaling based on one bit power control commands in the primary network is utilized to adjust the transmit power in the secondary network.

- The proposed multi time scale power allocation exploits both slow shadowing and fast fading to maximize the achievable rate of the secondary users.

- A comprehensive performance evaluation has been developed for the proposed power allocation in which errors that might happen in overhearing the primary service power control command are appropriately taken into account and the associated negative impacts on the performance of the primary and secondary service are analyzed.

\section{Organization of the Paper}

In Section II, the system model is presented. In Section III, we present and model the underlay spectrum sharing in DS-CDMA primary network. Then, in Section IV we present AMTPA method and investigate its performance. Practical considerations regarding the primary network, the probe user and power control feasibility in the primary network are also discussed in Section V. Simulation results are presented in Section VI, followed by conclusions in Section VII.

\section{SyStem Model}

Two services try to access the spectrum band: Primary Service and Secondary Service. The concerned frequency band is licensed to the primary service which we refer to as 'spectrum'. The primary network is a cellular DS-CDMA system with processing gain $G$ (see, e.g., [16]). The secondary service does not have the spectrum license, but may access the

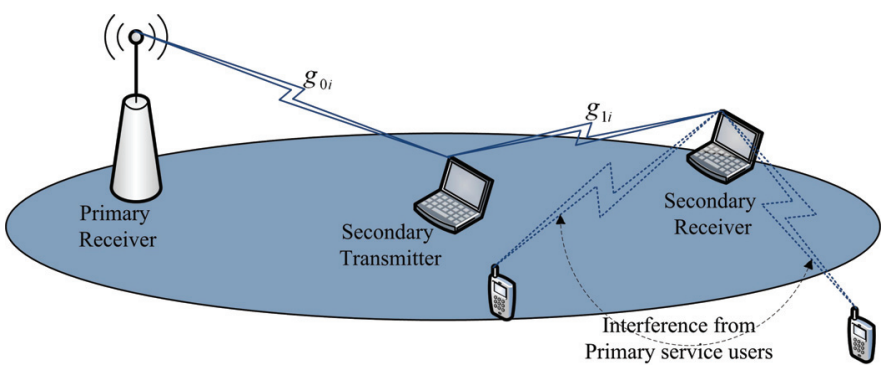

Fig. 1. System schematic for a sub-channel $i$.

spectrum by adopting underlay spectrum sharing. Subscripts $s$ and $p$ refer to the secondary service and the primary service, respectively. We define $I_{p \mid s}\left(I_{s \mid p}\right)$ as the interference created by the activity of the secondary service (primary service) at the primary (secondary) receiver.

We consider a frequency-selective wireless channel with additive white Gaussian noise (AWGN). The white noise power spectral density is $N_{0}$. From the secondary service viewpoint, the channel is divided into $N$ flat fading $B_{c} \mathrm{~Hz}$ subchannels, where $B_{c}$ is much smaller than the channel coherence bandwidth. Therefore, the total bandwidth is $B=N B_{c}$. Sub-channels are indexed by $i, i=1,2, \ldots, N$. The wireless channel also experiences a log-normal shadowing with zero mean and standard deviation of $\sigma_{d B} \mathrm{~dB}$. The coherence bandwidth of shadowing is usually much larger than that of flat fading; therefore, we assume that shadowing is constant for the whole bandwidth.

An schematic of the considered system in this paper is depicted in Fig. 1. For sub-channel $i, g_{0 i}$ and $g_{1 i}$ in Fig. 1 denote the instantaneous gains of sub-channel $i$ from the secondary transmitter to the primary receiver (i.e., crosssub-channel) and the secondary receiver (i.e., secondary-subchannel), respectively. Both $g_{0 i}$ and $g_{1 i}$ are assumed to be independent random variables with probability density functions (pdf), $f_{0 i}\left(g_{0 i}\right), f_{1 i}\left(g_{1 i}\right)$, respectively. Both $g_{0 i}$ and $g_{1 i}$ are i.i.d. for $i=1,2, \ldots, N$.

Time is divided into frames, indexed by $n$, each with $T_{\text {frame }}$ seconds duration. Frame duration $T_{\text {frame }}$ is much smaller than that of the shadowing coherence time; therefore, during each frame, the shadowing gain is constant. Each frame consists of $N_{p}$ slots indexed by $k, k=1,2, \ldots, N_{p}$ each with $T_{\text {slot }}$ seconds duration. The duration of slots $T_{\text {slot }}$ is much smaller than that of the coherence time of channel fading.

Link level performance in the primary network is a function of the corresponding signal to interference plus noise ratio (SINR). To achieve reliable communication, the SINR, or equivalently the bit energy to the interference spectral density $\left(E_{b} / I_{0}\right)$ should be above a certain threshold, namely $\rho$. For maintaining the targeted $E_{b} / I_{0}$ as the link level performance threshold in DS-CDMA systems, a power control mechanism is usually adopted [16]. Power control commands are communicated to the transmitter in an appropriate time-scale. Based on the received power control command, the transmitter then adjusts its transmit power to maintain targeted threshold for the required quality of service. Therefore, $E_{b} / I_{0}$ for all users in the BS coverage area is managed to be larger than or equal to $\rho$, which is a system parameter.

Here, we assume a fixed-step power control (FSPC) algorithm. FSPC is widely used in cellular DS-CDMA standards 
such as UMTS and IS-95 systems due to its simplicity and stability [17], [18]. The time-scale of FSPC is $T_{\text {slot }}$, i.e., the power control commands are transmitted each $T_{\text {slot }}$ seconds. In FSPC, the BS measures the corresponding received $E_{b} / I_{0}$ to an active mobile user and then compares it with the threshold, $\rho$. A one-bit power control command, $\Gamma_{p m}(n, k) \in\{0,1\}$, is then sent to the mobile station $m$ in the $k$ th slot of the $n$th frame. If $\Gamma_{p m}(n, k)=1\left(\Gamma_{p m}(n, k)=0\right)$ it indicates that the received $E_{b} / I_{0}$ is less (greater) than $\rho$ thus the mobile should increase (decrease) its transmit power by $\Delta_{p}$ Watts.

\section{Underlay SPECTRUM SHARING IN DS-CDMA PRIMARY NETWORK}

Let $M$ be the number of active primary users in the uplink of a DS-CDMA cellular network indexed by $m, m=$ $1,2, \ldots, M$. Without spectrum sharing, $E_{b} / I_{0}$ requirement for this system is satisfied if [15]

$$
E_{b} / I_{0}=\frac{G Q_{m}}{\sum_{j \neq m} Q_{j}+N_{0} B} \geq \rho, \quad m=1,2, \ldots, M
$$

where $G$ is the processing gain of DS-CDMA system, $Q_{j}$ is the received power corresponding to user $j$ at the front-end of the primary service receiver (i.e., BS), and $\rho$ is the $E_{b} / I_{0}$ threshold.

As the performance measure, we consider the outage probability which is defined as

$$
P_{\text {out }} \triangleq \operatorname{Pr}\left\{\frac{E_{b}}{I_{0}}<\rho\right\}
$$

where $\operatorname{Pr}\{A\}$ is the probability of occurrence of event $A$. In the ideal case, where $P_{\text {out }}=0$, the maximum number of active users in the primary network is [15]

$$
M_{0}=\frac{G}{\rho}+1
$$

If $P_{\text {out }}>0$, then the number of active users in the network coverage area may exceed $M_{0}$.

For a large number of users in DS-CDMA networks, invoking the Central Limit Theorem justifies the Gaussian approximation for the interference process [16]. Using secondorder statistics, it is shown that the interference process is also white [15]. In practice, the above argument is shown to be valid for a moderate number of users (e.g., 10-30) [19], [20]. Moreover, due to the fundamental limitation of the conventional DS-CDMA receivers (see e.g., [16]) the interference level in the receiver should not be increased beyond a threshold which is the product of the background noise power spectral density and a constant coefficient, which is a system parameter. Therefore, in DS-CDMA networks, the total received interference caused by other active users in the network coverage area is simply modelled as an additive white Gaussian noise. Moreover, the power spectral density of the received interference is equal to the product of the background noise power spectral density and a factor $(K-1)$ [15], [21]. In the following we obtain $K$.

In DS-CDMA network for each transmitter, a silence period detector is usually utilized to exploit the silence periods by stopping transmission during such periods. This technique reduces the interference thus results in a capacity gain. To model the user's activity in DS-CDMA network, users' activity factor, $\nu$, is defined that is the fraction of time in which the user is transmitting (see, e.g., [15], [22]). The more activity in the primary network, the higher the value of total interference is. Using the results presented in our previous works ([5], [22]), $K$ can be obtained for the uplink of a DSCDMA network with chip rate $W$, providing $S$ services each with $n_{s}$ users and activity factor $\nu_{s}$, required bit-energy to interference plus noise spectral density, $\rho_{s}$, and bit-rate $R_{s}$ as (see Appendix I)

$$
K=\left(1-(1+f) \sum_{s=1}^{S} n_{s}\left(1+\frac{B}{\rho_{s} \nu_{s} R_{s}}\right)^{-1}\right)^{-1} .
$$

In (4), $f$ is the ratio of the interference originated in the other cells to the interference generated in the home-cell which is a system parameter and is mainly a function of users' spatial distribution in the network coverage area [16]. As it is seen, for a given number of users in each service type (i.e., given $\left.n_{s}, s=1, \ldots, S\right), K$ in (4) is an increasing function of the users' activity. It is also observed in (4) that for a given set of services (i.e., given $\nu_{s}, s=1, \ldots, S$ ), $K$ is an increasing function of the number of users in the network coverage area.

In the case of a single cell single service network, or where there is no other-cell interference at the BS (i.e., $f=0, S=1$, $\left.n_{s}=M, \nu_{s}=1\right), K$ is obtained from (4)

$$
K=\frac{M_{0}}{M_{0}-M},
$$

where $M_{0}$ is defined in (3). Note that $K$ is only a function of the number of the active primary service users, $M$.

Consequently, similar to the primary users, for a secondary user that utilizes a $B_{c} \mathrm{~Hz}$ sub-channel and is located in the coverage area of the primary network, the average interference imposed by the primary network at the receiver can be approximated by

$$
I_{s \mid p}=(\tilde{K}-1) N_{0} B_{c}
$$

where $\tilde{K}$ is derived in [23].

In the following, for brevity of expositions, we assume that the secondary user accesses over only one sub-channel. If the secondary service access sub-channel $i$, a narrow-band interference, $I_{i, p \mid s}$, is correspondingly created at the receiver of the primary service (i.e., BS). The created interference $I_{i, p \mid s}$ may affect the performance of the primary system.

Here, we define Perfect Underlay Spectrum Sharing.

Definition (Perfect Underlay Spectrum Sharing): A perfect underlay spectrum sharing over sub-channel $i$ does not change $P_{\text {out }}$ in the primary network.

The following proposition gives a condition on $I_{i, p \mid s}$ that results in perfect underlay spectrum sharing.

Proposition 1: A perfect underlay spectrum sharing over sub-channel $i$ exists if

$$
I_{i, p \mid s} \leq N_{0} B\left(M_{0}-M-1\right)^{+} \triangleq Q_{I},
$$

where $(x)^{+}=\max \{0, x\}$.

Proof: Without underlay spectrum sharing, $E_{b} / I_{0}$ constraint in (1) is $\frac{G Q_{m}}{\sum_{j \neq m} Q_{j}+N_{0} B} \geq \rho$, where the equality is held if 
all the primary users $j, j=1, \ldots, M$ set their transmission powers such that the received power at the BS meets

$$
Q_{j}=\frac{N_{0} B}{M-M_{0}}, \quad M<M_{0} .
$$

For the case of underlay spectrum sharing, due the secondary service transmission there is an extra narrow-band interference power $I_{i, p \mid s}$ imposed at the BS. However, the primary service is already able to provide service to $M_{0}-1$ primary users. Consequently, one can assign the maximum value of $I_{i, p \mid s}$, i.e., $Q_{I}$, such that the primary service acts similar to the case of serving $M_{0}-1$ users instead of actual $M$ users as

$$
\frac{N_{0} B}{M_{0}-M_{0}+1}=\frac{N_{0} B+Q_{I}}{M_{0}-M}
$$

Obtaining $Q_{I}$ from (9) results in (7).

Proposition 1 indicates that if the secondary service activity causes $I_{i, p \mid s}$ to be greater than $Q_{I}$, then $E_{b} / I_{0}$ constraint is violated. Therefore, an outage incident is experienced. In such cases, the outage is a direct consequence of collision between the secondary service and the primary service transmissions.

For perfect underlay spectrum sharing, Proposition 1 suggests that the secondary service should adjust its transmission power to keep $I_{i, p \mid s} \leq Q_{I}$ at the receiver of the primary service. Therefore, the secondary transmitter requires the actual value of $I_{i, p \mid s}$ as well as $g_{0 i}$ to adjust its transmission power.

In practice, a direct signaling between two systems should be required for communicating the measured value of $I_{i, p \mid s}$ in the primary service, as well as $g_{0 i}$, to the secondary service transmitter. Moreover, the primary network is either not able, or not particularly designed to measure and send back $I_{i, p \mid s}$ and $g_{0 i}$ to the secondary service. Therefore, the secondary service has no direct means to estimate these two parameters.

\section{A. Outage and Collision}

Here, for brevity, in the primary network the outage caused by collision is disjointed from the outages caused by other non-spectrum-sharing related effects. In other words, hereafter we consider collision as an outage exclusively caused by the secondary service activity. Therefore, the collision probability $P_{\text {col }(i)}$ is a metric that indicates the impact of underlay spectrum sharing over sub-channel $i$ on the primary service. The acceptable level of collision probability, $\xi$, is defined by

$$
P_{\text {col }(i)}=\operatorname{Pr}\left\{I_{i, p \mid s}>Q_{I}\right\} \leq \xi .
$$

Generally, $\xi$ is a system parameter set by either the primary service operator, or the spectrum sharing regulator. Thus, underlay spectrum sharing should act in a way that the collision probability in the primary network is always less than or equal to $\xi$. Hereafter, we refer to $\operatorname{Pr}\left\{I_{i, p \mid s}>Q_{I}\right\}$ as the collision probability.

\section{B. Primary Network Power Control and Collision}

In power controlled DS-CDMA, BS routinely reacts to the violations of $E_{b} / I_{0}$ constraint. Different parameters in the primary network may usually cause $E_{b} / I_{0}$ constraint to be violated. Having the secondary service active in the primary network, the power control mechanism also reacts to the violations of $E_{b} / I_{0}$ directly caused by the secondary

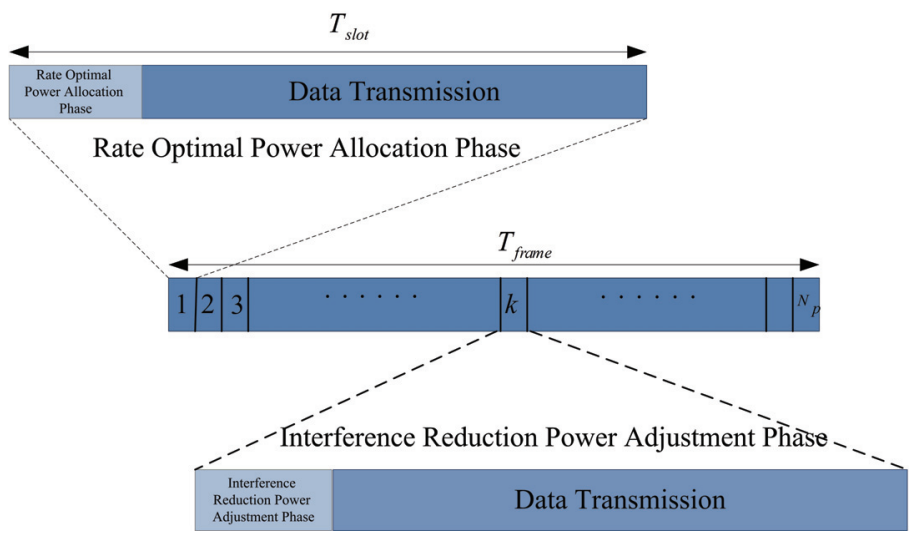

Fig. 2. Time is divided into frames (namely AMTPA frames), each frame consists of a number of slots, $N_{p}$. In the first slot of the frame, rate optimal power allocation phase is performed; in the consequent slots of the frame, interference reduction power adjustment phase is conducted.

service activity (i.e., correspondingly the violation of (7)). The reaction is to send appropriate power control commands to the affected primary service users. Thus, the power control commands, in a way, contain information on and about the interference level at the receiver of the primary service. Listening to the power control commands, the secondary user might be able to adjust its transmission power so that (7) is held. In the following, we present a method which exploits power control commands transmitted by the BS to the primary users, to adjust the power levels of the secondary users.

\section{AMTPA}

The main objective of power allocation in the secondary service is to adjust the secondary service transmission power so that no quality degradation is imposed on the active primary service users. To achieve this objective, the values of $I_{i, p \mid s}$, and $g_{0 i}$ are required by the secondary service to set its transmission power, so that Proposition 1 is held. However, as it was mentioned previously, considering such signaling framework is not always attainable. To tackle this issue, we propose the Adaptive Multiple Time-scale Power Allocation (AMTPA) which exploits power control commands and does not require direct signaling between the primary and secondary systems.

AMTPA is a power allocation strategy adopted in the secondary service transmitter which consists of two phases in different time-scales (Fig. 2): Rate Optimal Power Allocation and Interference Reduction Power Adjustment. In the longer time scale, $T_{\text {frame }}$, (i.e., at the beginning of each frame), the rate optimal power allocation phase allocates the transmit power of the secondary service transmitter with the objective of maximizing the achievable capacity of the secondary channel and without considering $Q_{I}$ in (7). The rate optimal power allocation phase may cause an increase in the received interference in the primary network; thus, results in decreasing $E_{b} / I_{0}$ and consequently increasing bit-error-rate, i.e., QoS degradation. Then, in the shorter time-scale, $T_{\text {slot }}$, the interference reduction power adjustment phase tries to reduce the QoS degradation through implementing the primary service interference constraint in (7) by adjusting the allocated transmit power of the secondary service. 


\section{A. Rate Optimal Power Allocation Phase}

In the rate optimal power allocation phase which is over the longer time-scale, we assume that only shadowing effect is involved. This assumption can be justified by adopting Jensen's inequality as follows. The ergodic capacity of the secondary service over sub-channel $i, C_{s i}$, is

$$
C_{s i} \leq B_{c} \mathbf{E}_{\text {shad }}\left\{\log \left(1+\frac{g_{1 i} P_{s i}}{\tilde{K} N_{0} B_{c}}\right)\right\},
$$

where $\mathbf{E}_{\text {shad }}$ indicate expectations over shadowing. Consequently, in (11), $g_{1 i}$ only contains shadowing effect. The optimal secondary service power allocation over sub-channel $i$ in frame $n$ is then obtained via water filling (WF) algorithm [24] as

$$
P_{s i, W F}(n)= \begin{cases}\frac{1}{\lambda_{0}}-\frac{\tilde{K} N_{0} B_{c}}{g_{1 i}(n)} & \text { if } g_{1 i}(n) \geq \lambda_{0} \tilde{K} N_{0} B_{c} \\ 0 & \text { O.W. }\end{cases}
$$

where $\lambda_{0}$ is the Lagrangian coefficient adjusted to meet the transmission power constraint of the secondary service, $\bar{P}_{s, \max }$.

Finding sub-channel $i$ itself, is an optimization problem which can be conducted with the objective which is a combination of imposing minimum interference over the primary service and achieving maximum capacity in the secondary system. Solving such optimization problem is elaborated in one of our previous works [25] in which both secondary and cross channels are required. However, since the cross channel is assumed to be unavailable, for brevity of expositions, we simply assume that the sub-channel in rate optimal power allocation phase is randomly selected. Obviously, adopting one of the optimal sub-channel selection scenarios in [25] a higher capacity is achieved in the rate optimal power allocation phase.

In AMTPA, the secondary service transmit power at the first slot of the $n$th frame, i.e., $P_{s i}(n, 1)$, is set as the output of the rate optimal power allocation phase, i.e., $P_{s i, W F}(n)$,

$$
P_{s i}(n, 1)=P_{s i, W F}(n),
$$

where $P_{s i}(n, k)$ is the transmit power of the secondary service user at slot $k$ in frame $n$ of sub-channel $i$. Employing only the rate optimal power allocation phase, the secondary service transmit power during frame $n$ remains equal to $P_{s i, W F}(n)$. This allocated power imposes an equivalent narrow-band interference, i.e., $I_{i, p \mid s}(n)$, to the receiver of the primary service ${ }^{1}$

$$
I_{i, p \mid s}(n)=g_{0 i}(n) P_{s i, W F}(n) .
$$

Thus if $I_{i, p \mid s}(n)>Q_{I}$, a collision is occurred. The following proposition provides the probability of collision caused by the rate optimal power allocation phase in the primary network.

Proposition 2: If both $g_{0 i}$ and $g_{1 i}$ are log-normal shadowing with standard deviation $\sigma_{d B}$, the probability of collision caused by performing rate optimal power allocation phase over

\footnotetext{
${ }^{1}$ Unless otherwise specified, it is assumed that the effect of distance dependent path-loss attenuation is scaled in the background noise power, and interference threshold $Q_{I}$, at the secondary, and primary service receivers, respectively.
}

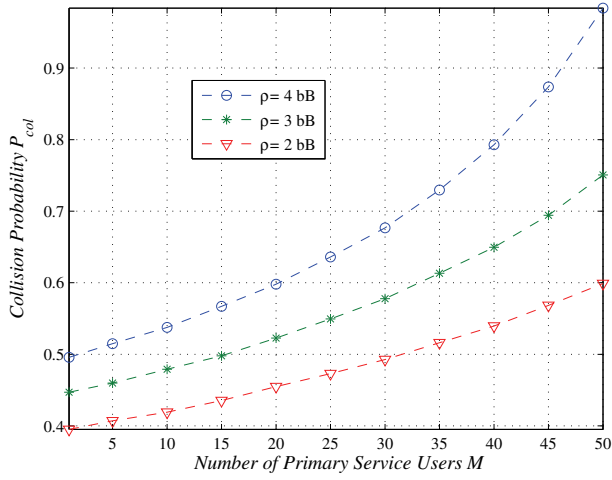

Fig. 3. Collision probability versus $M$ in the rate optimal power allocation phase, $\left(G=128, \bar{P}_{s, \max }=1 \mathrm{Watt}\right.$, and $\left.\sigma_{d B}=8 \mathrm{~dB}\right)$.

a sub-channel $i$ is

$$
P_{\operatorname{col}(i)}=\frac{1}{2} \int_{\boldsymbol{\Phi}} \operatorname{erfc}\left(\frac{\ln \left[\frac{Q_{I}}{\left(\frac{1}{\lambda_{0}}-\frac{K N_{0} B_{c}}{\alpha}\right)}\right]}{\sqrt{2} \sigma}\right) f_{1 i}(\alpha) d \alpha,
$$

where, $\boldsymbol{\Phi} \triangleq\left\{\alpha \mid \alpha \geq \lambda_{0} \tilde{K} N_{0} B_{c}\right\}, \operatorname{erfc}(x) \triangleq \frac{2}{\sqrt{\pi}} \int_{x}^{\infty} e^{-t^{2}} d t$, $f_{1 i}(x)=\frac{1}{x \sigma \sqrt{2 \pi}} \exp \left(-\frac{(\ln x)^{2}}{2 \sigma^{2}}\right)$, and $\sigma=0.1 \log _{10} \sigma_{d B}$.

Proof: See Appendix II.

In Fig. 3, $P_{\operatorname{col}(i)}$ is plotted versus number of the primary service users, $M$, for a randomly selected sub-channel $i$ considering $G=128$, where the standard deviation of lognormal shadowing, $\sigma_{d B}$, is $8 \mathrm{~dB}$. According to Fig. 3, by increasing $M$, the probability of collision is increased since the amount of $Q_{I}$ is decreased. Note that by increasing $\rho$, the probability of collision for a given $M$ increases, because $Q_{I}$ decreases.

The rate optimal power allocation phase does not consider $Q_{I}$ and small-scale variations of wireless channel caused by fast fading. Therefore, the secondary service activity based on the allocated power in the rate optimal power allocation phase may cause collision and have destructive impact on the performance of the primary network. Interference reduction power adjustment phase in AMTPA has been designed to decrease the impact of rate optimal power allocation phase through adaptive adjustment of the transmission power in the shorter time-scales, i.e., $T_{\text {slot }}$.

\section{B. Interference Reduction Power Adjustment Phase}

Interference reduction power adjustment phase adaptively adjusts transmission power by exploiting the power control commands transmitted by the BS into the probe user. Probe user is a primary user $m$ which is selected and associated with the secondary service either randomly, or by the primary network. In AMTPA, we set $P_{s i}(n, 1)=P_{s i, W F}(n)$, for slot 1 in frame $n$. Then, the interference reduction power adjustment phase, adjusts the transmit power based on $\Gamma_{p m}(n, k)$ so that the transmission power of the secondary service is decreased 


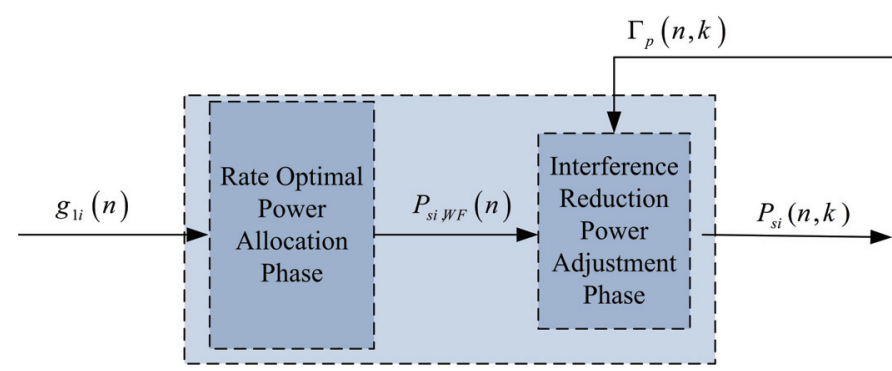

Fig. 4. AMTPA block diagram.

(increased) by $\Delta_{s}$ if $\Gamma_{p m}(n, k)=1\left(\Gamma_{p m}(n, k)=0\right)$, i.e.,

$$
P_{s i}(n, k)=\left(P_{s i}(n, k-1)+\Delta_{s}\left(1-2 \Gamma_{p m}(n, k)\right)\right)^{+} \text {. }
$$

This is illustrated in Fig. 4.

Note that, after receiving power control commands, the secondary service takes action based on a pessimistic strategy presented in (16). In (16), it is implicitly assumed that any increase in the interference received by the primary user is exclusively caused by the secondary user. This argument emanates from the fact that the secondary service is not the licensee; thus should release the spectrum immediately, if it is required by the the primary service.

After applying the interference reduction power adjustment phase, $E_{b} / I_{0}$ requirement within frame $n$ for user $m$, i.e., (1), is modified as

$$
\frac{G Q_{m}}{\sum_{j \neq m} Q_{j}+I_{i, p \mid s}(n, k)+N_{0} B} \geq \rho, k=1, \ldots, N_{p},
$$

where $I_{i, p \mid s}(n, k)$ is the narrow-band interference caused through applying (16) in the secondary transmitter. If for any reason (17) is violated at slot $k$, a power control command $\Gamma_{p m}(n, k+1)=1$ is issued by the primary BS to the primary user to increase its transmission power by $\Delta_{p}$ Watts. After receiving this command, at the same time the secondary user reduces its transmission power by $\Delta_{s}$ Watts.

\section{Modified-AMTPA}

In the interference reduction power adjustment phase, setting $P_{s i}(n, 1)=P_{s i, W F}(n)$ causes the system history associated with $P_{s i}\left(n-1, N_{p}\right)$, not to be incorporated into the interference reduction power adjustment phase in the subsequent slots. To incorporate the "system history" we propose the Modified-AMTPA, in which the interference reduction power adjustment phase is modified as

$$
P_{s i}(n, 1)=\min \left\{P_{s i, W F}(n), P_{s i}\left(n-1, N_{p}\right)\right\},
$$

where $P_{s i, W F}(n)$ is obtained by (12) and $P_{s i}\left(n-1, N_{p}\right)$ is the transmitted power of the last slot of the previous frame i.e., $n-1$. Eq. (18) makes the power assignment for the first slot of each frame in favor of the primary service. Applying this modification, we expect the number of collisions and $P_{\operatorname{col}(i)}$ to decrease. This claim is verified through simulations in Section VI.

\section{Random-Modified-AMTPA}

In addition to the operation of the secondary service, there might be other reasons for the $\mathrm{BS}$ to issue a power control command, e.g., temporal variations of the wireless channel between the BS and the primary user and/or interference fluctuation in the primary network. However, in the interference reduction power adjustment phase we assume that the secondary service is the only reason for issuing a power control command. This is the reason we called the power allocation strategy in (16) a "pessimistic strategy" that particularly acts in favor of the primary network.

Moreover, in practice, to reduce delay and overhead, the power control commands are transmitted without error correcting codes. Therefore, approximately $10 \%$ of the power control command are received erroneous [26]. Let $p_{\text {error }}$ be the probability of existence of error in the received power control command. The error can be modelled by multiplying $2 \Gamma_{p m}(n, k)-1$ by $1-2 E_{p}(n, k)$, where $E_{p}(n, k)$ is 1 with probability $p_{\text {error }}$ (error occurs), and 0 with probability $1-p_{\text {error }}$ (error does not occur), otherwise.

To incorporate the above concerns, we propose the RandomModified-AMTPA. In this method, (18) used for $P_{s i}(n, 1)$, as it was in the Modified-AMTPA. However, the interference reduction power adjustment phase in the Random-ModifiedAMTPA takes the power control command into consideration with a probability of $p_{R M}$, where $p_{R M}$ is a system parameter that can be adjusted by experiment. In this approach, if $\Gamma_{p m}(n, k)=1\left(\Gamma_{p m}(n, k)=0\right)$, then the transmission power of the secondary service is decreased (increased) by the probability $p_{R M}$ and remains the same with the probability of $1-p_{R M}$.

\section{E. Secondary Service Power Control}

In cases where the secondary service has its own power control, the interference reduction power adjustment phase in (16) can be then conservatively modified as follows:

$$
\begin{gathered}
P_{s i}(n, k)=\left(P_{s i}(n, k-1)+\Delta_{s}(1-2 \mathcal{T}(n, k))\right)^{+}, \\
\mathcal{T}(n, k)=\Gamma_{(s) s}(n, k) \vee \Gamma_{p m}(n, k),
\end{gathered}
$$

where " $\vee$ " represents logical OR, and $\Gamma_{(s) s}(n, k)$ represents the power control command issued by the receiver of the secondary service in slot $k$ of frame $n, \Gamma_{(s) s}(n, k)=1$ $\left(\Gamma_{(s) s}(n, k)=0\right)$ corresponds to a decrease (increase) in the transmit power of the secondary user. It is worth mentioning that $\Gamma_{(s) s}(n, k)=1$, means that the received signal at the secondary receiver has enough power to be decoded correctly.

\section{F. Enforcing Primary Service Collision Probability Constraint}

Utilizing Proposition 2, for a given collision probability in the primary network, $\xi$, we can obtain the corresponding $Q_{I}$. Using (14) the corresponding transmit power of the secondary service over sub-channel $i$ in slot 1 of frame $n$ is obtained using (13) as

$$
P_{s i}(n, 1)=\min \left\{P_{s i, W F}(n), \frac{Q_{I}}{g_{0 i}(n)}\right\} .
$$

We refer to this version as AMTPA with collision probability constraint enforcing (AMTPA-CPE). In cases where the secondary service knows the transmit power of the $\mathrm{SCH}$ channel (see Section V-A for a brief discussion on $\mathrm{SCH}$ ) in the primary network, $g_{0 i}(n)$ can be easily obtained assuming 
reciprocity. Note that, in this case an estimate of $g_{0 i}$ is obtained in the shadowing time scale and still no signaling is required between the primary and secondary systems.

\section{Practical Considerations}

\section{A. Primary Network}

In DS-CDMA networks, a synchronization channel (SCH) is broadcasted by the $\mathrm{BS}$ to establish the coverage area of a cell [21]. The SCH also includes the cell-id and other necessary information for users to get connected to the network through the corresponding BS. In AMTPA, secondary users should utilize the information contained in the $\mathrm{SCH}$, thus should be facilitated by the ability of receiving and decoding the $\mathrm{SCH}$ information.

\section{B. The Probe User}

The probe user is either selected randomly or associated with the secondary service by the primary network. It can be also set permanently by the primary network operator. Accordingly, the right to access the power control signaling of the probe user should be granted to the secondary service. Therefore, an entity in the primary network should provide the secondary user with the required information to receive and decode the power control command issued by the primary network to the probe user. Consequently, there should be a cooperation framework in higher layers between the two systems.

Actually, it could be possible for the primary network to consider a virtual probe user. In such scenario, the primary service BS generates a specific set of power control command for the secondary service based on the primary network situation. Transmitting power control commands acts as the signaling between the primary and secondary services. Therefore, in such case, AMTPA could act upon the received power control commands. We expect that in this case, AMPTA performance is significantly increased. However, one can say such scenario increases the complexity of the primary network and may not be attainable in practice.

\section{Primary Network Stability and Feasibility}

Practically in power controlled systems, there may be cases where, due to lack of enough radio resources, it is infeasible for the power control mechanism to satisfy the required $E_{b} / I_{0}$ for all active users at the same time. This situation is sometimes referred to as power control instability or infeasibility [27], [28]. In such cases, one solution is to adopt an appropriate removal strategy, which removes some active users, so that the power control becomes stable for the rest of the users [27], [28].

In AMTPA, the secondary service activity may cause the power control mechanism in the primary network to become instable. We note that the necessary condition for feasibility of the power control algorithm is that the spectral radius ${ }^{2}$ of matrix $\tilde{\mathbf{H}}$ should be equal or less than 1 , where the elements of $\tilde{\mathbf{H}}$ are the primary channel gains. If the power

\footnotetext{
${ }^{2}$ The spectral radius of matrix $\mathbf{A}$ with eigenvalues $e_{1}, e_{2}, \ldots$, is defined as $\operatorname{Spec}(\mathbf{A}) \triangleq \max _{j} e_{j}$.
}

of background noise at the BS is zero; therefore, the spectral radius of $\tilde{\mathbf{H}}$ is equal to 1 . However, based on a nonzero noise power, an infinite transmission power of the primary service transmitters is required. Although in practice, the primary service transmitters have usually some constraints on their transmission powers. Therefore, for some finite number of the primary service users (e.g., $M<M_{0}$ ) the power control algorithm is feasible and a unique power vector can be obtained.

In case, where a secondary service accesses to the spectrum, matrix $\tilde{\mathbf{H}}$ remains unchanged. Therefore, the necessary condition for feasibility of the power control is not changed. However, increasing the interference at the primary service receiver may make the power control algorithm in the primary service infeasible. In this case, given that the secondary service is able to adjust its transmission power based on (7), we expect that for suitable $\Delta_{s}$ the primary service power control remains feasible, although optimal power vector is modified. Indeed, holding (7) enforces the DS-CDMA system with $M<M_{0}$ active primary service users to act as a system with $M$ active users with the equivalent interference generated by $M_{0}$ active users. Since, the power control is already feasible for supporting up to $M_{0}$ active users, it remains feasible.

We can show that the primary network power control is feasible and using AMTPA-CPE, there is a region for $\Delta_{s}$ in which the power control in the primary network remains feasible. The obtained upper bound of $\Delta_{s}$ suggests us how to select the probe user. Using this upper bound, the BS can select the probe user based on the values of the channel power gains among the BS and the primary users, and eigenvalues of the primary users channel matrix.

As it was mentioned before, to maintain power control feasibility usually a removal strategy is adopted by the primary network. In such a case, a straightforward strategy would be a removal strategy so that the first priority for removal is the active secondary service users. In AMTPA we consider the following secondary service dropping strategy along with the removal strategy in the primary network. The secondary service transmitter stops transmitting if it receives $k_{D}$ consequent reduce power control commands. System parameter $k_{D}$ is selected by the primary service operator.

\section{Simulation Results}

We simulate a multiple secondary service spectrum sharing system including a primary cellular DS-CDMA network and a single secondary access point with 10 randomly selected subchannels (see, Fig. 5). The wireless channel model consists of log-normal shadowing with a Rayleigh fast fading.

The primary network is a single cell DS-CDMA network with 2000 meter radius. Primary users are uniformly distributed in the coverage area. The system bandwidth is 5 $\mathrm{MHz}$. A fast power control mechanism is performed in the primary network in which the BS transmits 1500 power control commands per second.

The secondary services utilized AMTPA for underlay spectrum sharing in the uplink spectrum of the primary network for downlink transmission between the secondary service access point and the secondary service users. The bandwidth of each sub-channel is $80 \mathrm{KHz}$. The secondary access-point has a 


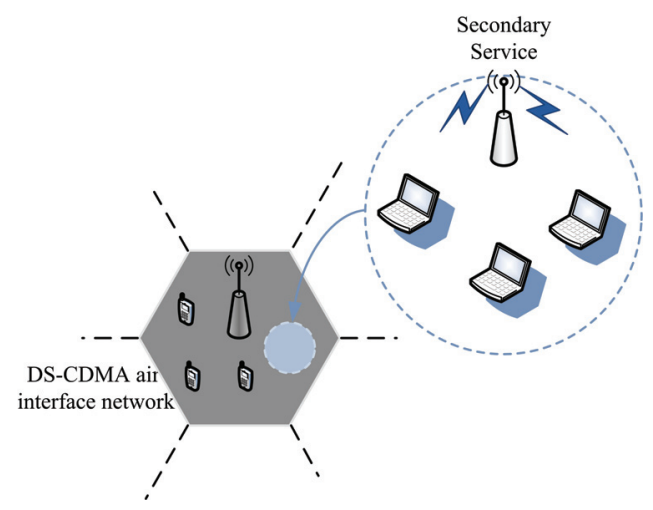

Fig. 5. The simulated spectrum sharing system with a DS-CDMA primary network and an OFDM-based secondary service access point.

TABLE I

SimUlation PARAMETERS

\begin{tabular}{lc}
\hline Parameter & Value \\
\hline$N_{p}$ & 32 \\
DS-CDMA cell radius & $2000 \mathrm{~m}$ \\
Secondary service cell radius & $500 \mathrm{~m}$ \\
Spectrum Bandwidth & $5 \mathrm{MHz}$ \\
Standard dev. of log-normal shadowing & $8 \mathrm{~dB}$ \\
Average of Rayleigh fading & 1 \\
Propagation loss exponent & 4 \\
Background noise power spectral density & $-174.0 \mathrm{dBm} / \mathrm{Hz}$ \\
$\bar{P}_{s, \text { max }}$ & $1 \mathrm{Watt}$ \\
$G$ & 128 \\
Number of secondary users & 10 \\
Number of sub-channels $N$ & 64 (each $80 \mathrm{KHz})$ \\
Minimum required $E_{b} / I_{0}$ (primary network) & $2 \mathrm{~dB}$ \\
$M_{0}^{\dagger}$ & $\simeq 81$ \\
Primary user power constraint & $1 \mathrm{Watt}$ \\
$\Delta_{p}$ & $0.1 \mathrm{Watt}$ \\
Distance between the secondary and & \\
primary BSs & $1000 \mathrm{~m}$ \\
\hline 'Parameter $M_{0}$ is obtained using (3).
\end{tabular}

circular coverage area with radius 500 meter. In each snapshot of the simulation the primary and secondary users are randomly located throughout the coverage area. The presented results is the average of the results from enough number of simulation snap-shots. Secondary service access point allocates its transmit power to the secondary service users in a way that they do not impose interference over each other. User $m$ (the probe user) is randomly selected by the primary service access point and associated with a secondary service.

Simulation parameters are presented in Table I. The main performance measures are $P_{c o l}$ (i.e., the probability of collision for selected sub-channels) and capacity of the secondary service which we study in the following considering different scenarios.

\section{A. Impact of $\Delta_{s}$}

To study the impact of $\Delta_{s}$, in the simulated system we obtain the collision probability of the primary service. The obtained collision probability is plotted versus $\Delta_{s}$ in Fig. 6 for different values of $M$. As it is seen, the probability of collision is decreased by increasing $M$. We elaborate on this later in this section. The observed pattern of the probability of collision variations is the same for different primary network loads (i.e., for different values of $M$ ). Note that $\Delta_{s}=0$ is

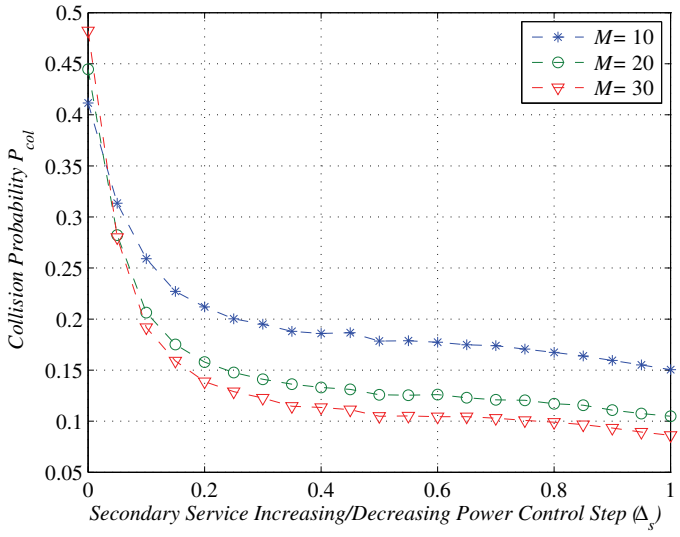

Fig. 6. Collision probability vs. $\Delta_{s}$ for $M=10,20,30$.

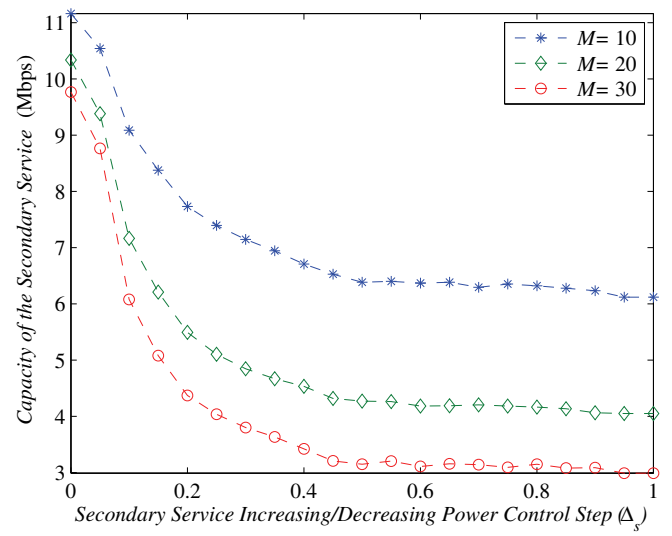

Fig. 7. Capacity of AMTPA vs. $\Delta_{s}$ for $M=10,20,30$.

equivalent to AMTPA without interference reduction power adjustment phase.

By increasing $\Delta_{s}$ from 0 to $\Delta_{s} \approx 0.4 \bar{P}_{s, \max }$, probability of collision is significantly decreased. However, for $0.4 \bar{P}_{s, \max } \leq \Delta_{s} \leq \bar{P}_{s, \max }$ it is seen that $P_{c o l}$ is not approximately changed. This is due to the fact that for larger values of $\Delta_{s}$ the secondary service user actually stops transmission after receiving a couple of decreasing power control commands. Therefore, the collision probability is not affected.

We also study the impact of $\Delta_{s}$ on the capacity of the secondary service for different values of $M$ in Fig. 7. As it is seen by increasing the number of the primary users, $M$, the capacity of the secondary service is decreased. Fig. 7 also indicates that by increasing $\Delta_{s}$ from 0 to $0.4 \bar{P}_{s, \max }$ the capacity of the secondary service is decreased. For $0.4 \bar{P}_{s, \max } \leq \Delta_{s} \leq \bar{P}_{s, \max }$ the capacity of the secondary service is approximately constant. As it is seen in the two above simulations $\Delta_{s}=0.4 \bar{P}_{s, \max }$ is appropriate for AMTPA. In the rest of our simulations we set $\Delta_{s}=0.4 \bar{P}_{s, \max }$.

We compared the impact of $\Delta_{s}$ on AMTPA and ModifiedAMTPA for a given value of $M=10$ in Fig. 8. It is observed that the probability of collision for a given value of $\Delta_{s}$ in Modified-AMTPA is smaller than that of AMTPA. Furthermore, the Modified-AMTPA is more sensitive to the choice of $\Delta_{s}$. Indeed, for $\Delta_{s} \approx 0.05 \bar{P}_{s, \max }$ the collision probability is minimum for Modified-AMTPA. For sufficiently large values of $\Delta_{s}$, i.e., $\Delta_{s} \geq 0.4 \bar{P}_{s, \max }$, both of AMTPA and ModifiedAMTPA follow the same patters and have the same collision 


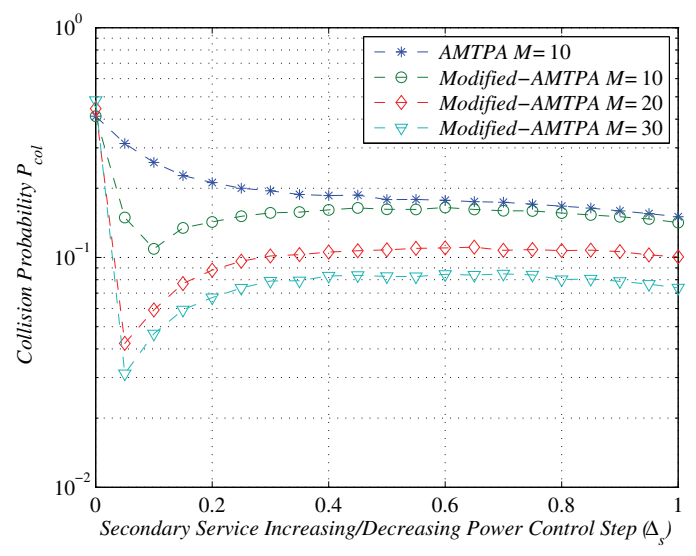

Fig. 8. Collision probability vs. $\Delta_{s}$ for AMTPA and Modified-AMTPA.

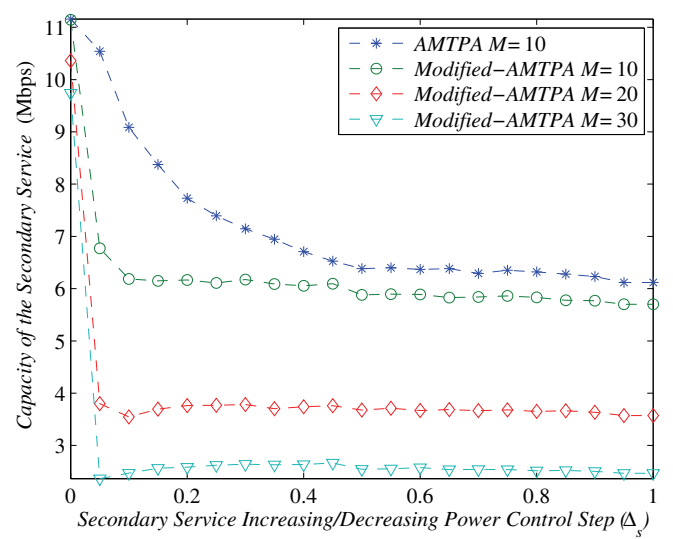

Fig. 9. Capacity of AMTPA and Modified-AMTPA vs. $\Delta_{s}$.

performances. This is mainly due to the following. Although in AMTPA a higher power level transmission is allocated to the secondary service in the start slot of the new frame in the contrary of Modified-AMTPA, in both AMTPA and ModifiedAMTPA the secondary service allocates a similar power level to the slots within each frame. Fig. 8 also presents the collision probability in Modified-AMTPA for different values of $M$. As it is seen, similar trend is observed in all cases; for $\Delta_{s} \approx 0.05 \bar{P}_{s, \max }$ the collision probability is minimum for Modified-AMTPA.

For small values of $\Delta_{s}$, the Modified-AMTPA outperforms the AMTPA from the collision probability viewpoint. The rapid decrease observed in the collision probability for the Modified-AMTPA by increasing Delt $a_{s}$ from zero to approximately $0.1 \bar{P}_{\text {max }}$, is due to the limiting effect of the secondary service transmission power in the first time slot of each frame. The limiting effect is because of the "min" operator which is implemented in the Modified-AMTPA. For moderate $\Delta_{s}$, it is seen that the collision probability of Modified-AMTPA approaches to the AMTPA collision performance. This is because the fact that for moderate and large $\Delta_{s}$ in some cases the secondary service is allocated a higher power level at the last time slot of the previous frame, comparing to the power allocated in the first slot of the new frame based on water-filling. For large values of $\Delta_{s}$ both of the AMTPA and Modified-AMTPA behave similarly in collision probability perspective.

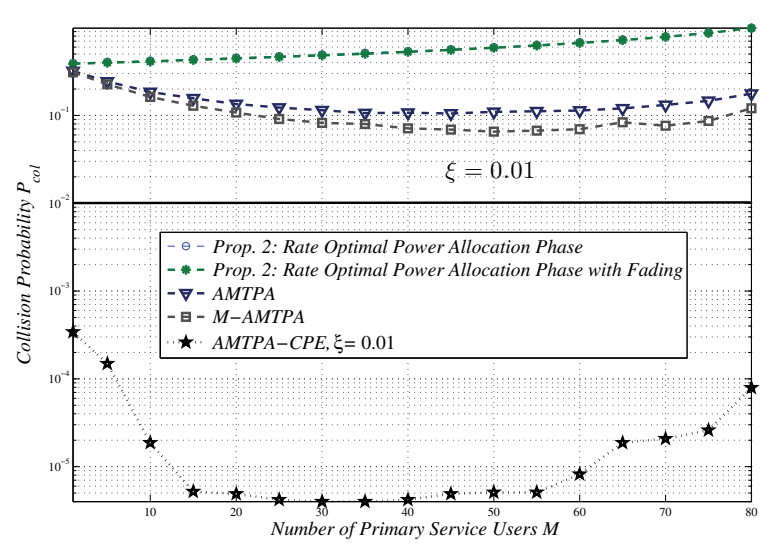

Fig. 10. Collision probability vs. $M$, for a system which only performs the rate optimal power allocation phase (AMTPA without power adjustment), and AMTPA variations.

In Fig. 9 we present the capacity of the secondary service versus $\Delta_{s}$ in AMTPA and Modified-AMTPA. As it is seen, the capacity of the Modified-AMTPA is smaller than that of AMTPA. However, similar to the pattern observed in Fig. 8, the Modified-AMTPA is more sensitive to the choice of $\Delta_{s}$. Putting the results in Fig. 8 together with Fig. 9 we conclude that increasing $M$, decreases the collision probability and the secondary service capacity. Noting Fig. 8 and Fig. 9 shows that the Modified-AMTPA achieves better collision probability but worse capacity performance comparing to the AMTPA.

The above simulations shows that one can select $\Delta_{s}$ with different objectives such as decreasing the collision probability, or increasing the capacity.

\section{B. Collision Probability}

We study the collision probability in Fig. 10. We consider the obtained collision probability for rate optimal power allocation phase in Proposition 2 under shadowing channel model as a benchmark. Then, we compare it with the simulation results when fading is also considered for AMTPA and Modified-AMTPA.

First, we simulate the rate optimal power allocation phase for a system with both shadowing (which has been also assumed in derivations of Proposition 2) and fading. As it is seen in Fig. 10, simulation results follow the obtained collision probability in (15) closely. This is mainly due to the fact that large enough number of fading events (with a time scale close to slot duration), is observed within a frame (i.e., $N_{p}=32$ ).

We then apply interference reduction power adjustment phase and conduct the simulation for the case where both fading and shadowing exist. Fig. 10 shows the corrective effect of the interference reduction power adjustment phase which significantly reduces the collision probability in comparison with AMTPA without interference reduction power adjustment phase. This result shows the effectiveness of interference reduction power adjustment phase in reducing the collision probability. We also observe that the gap between the collision probabilities in these two cases is increased by increasing $M$.

Fig. 10 also shows by increasing $M$ from 1 to roughly 60 the collision probability in AMTPA is reduced. This is because of increasing the rate of the power decreasing 


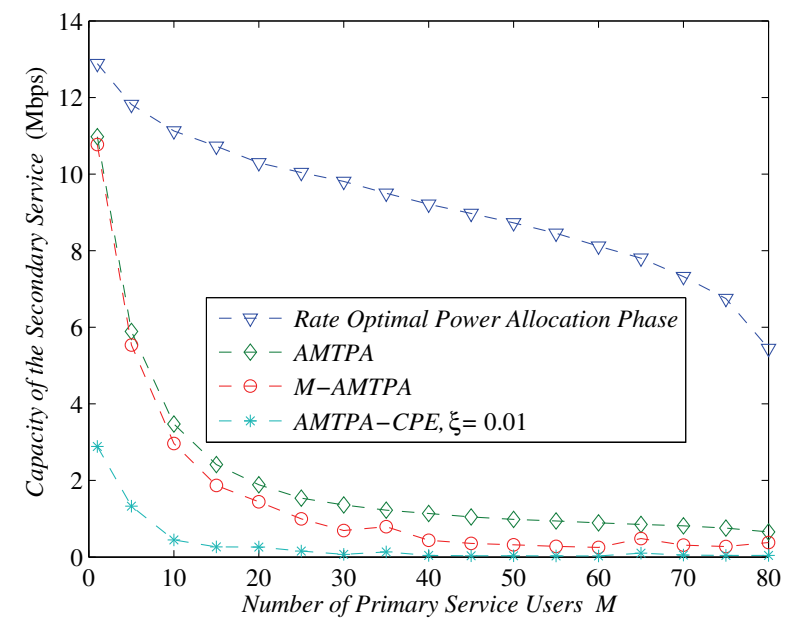

Fig. 11. Secondary service capacity $v s . M$, for a system which only performs the rate optimal power allocation phase (AMTPA without power adjustment) and a system which also performs the interference reduction power adjustment phase (AMTPA and its modifications).

commands issued by the primary service power control. For $60 \leq M \leq 80$, the collision probability is increased. In this case, on the one hand the amount of the interference threshold, $Q_{I}$, decreases and approaches to zero, and on the other hand, the rate of power reduction commands in the primary service power control is significantly increased. This results in rare power increase in the secondary service. However, even this rare event may result in collision because of small $Q_{I}$.

To show the impact of incorporating system history in AMTPA, we also compare the collision probability of AMTPA and that of Modified-AMTPA. Fig. 10 shows a slight improvement in the collision probability of Modified-AMTPA comparing to AMTPA.

We also simulate AMTPA with collision probability enforcing (AMTPA-CPE) for $\xi=0.01$ as it was presented in Section IV-F. As it is seen in Fig. 10 using AMTPA-CPE, the actual collision probability always satisfies the acceptable collision probability by the primary network i.e., $\xi=0.01$. This simulation shows that one can guarantee a certain level of collision probability in the primary network by conservatively selecting appropriate level of $Q_{I}$ and implying it into the allocation phase. Note that in AMTPA-CPE, the target collision probability $\xi$ is applied for the first time slot; thus, $p_{c o l}$ is smaller than the targeted value, even in the first time slot. However, employing interference reduction power adjustment phase further decreases the collision probability.

This simulation indicates that providing a certain outage probability in the primary network is possible, however the cost is decreasing secondary service capacity. To investigate this, simulate a spectrum sharing system in which the secondary service only performs the rate optimal power allocation phase (i.e., AMTPA without power adjustment) and compare its capacity with a system which also performs the interference reduction power adjustment phase (AMTPA and its modifications). The results are presented in Fig. 11. As it is seen, in the case of the rate optimal power allocation phase, increasing $M$ results in decreasing of the secondary service capacity. This is due to the fact that the received interference at the secondary service receivers imposed by the primary users is increased.

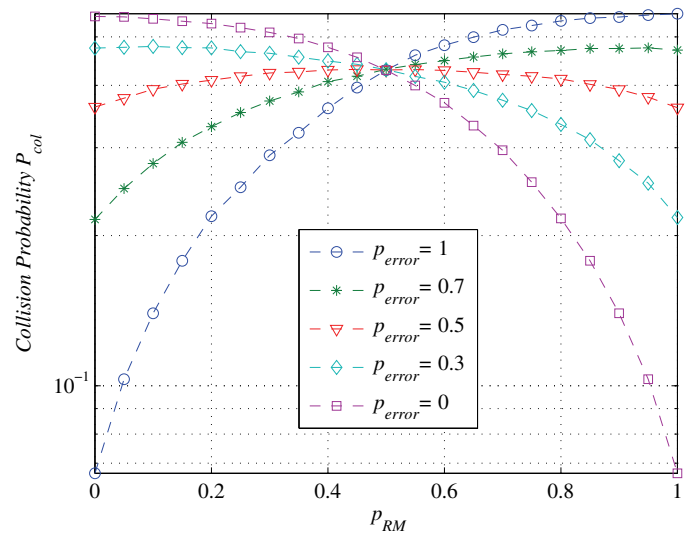

Fig. 12. Collision probability of Random-Modified-AMTPA vs. $P_{R M}$.

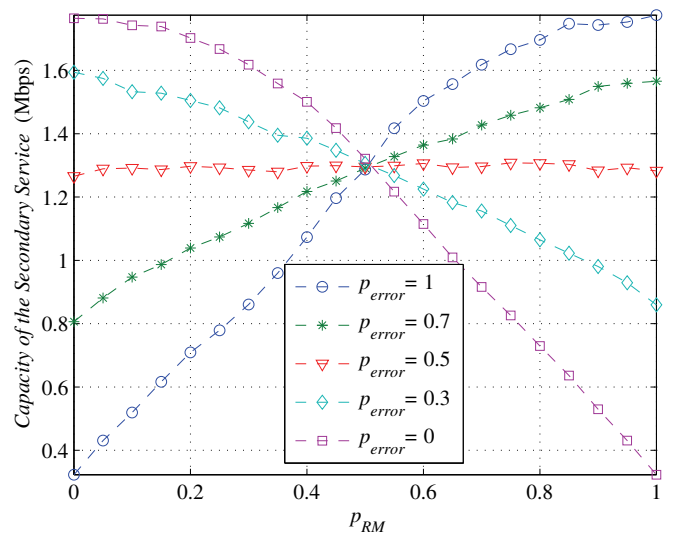

Fig. 13. Secondary service capacity of Random-Modified-AMTPA vs. $P_{R M}$.

Similar pattern is observed for AMTPA with the interference reduction power adjustment phase, Modified-AMTPA, and AMTPA-CPE. As it is seen, interference reduction power adjustment phase results in decreasing the secondary service capacity, comparing to the case where only the rate optimal power allocation phase is adopted. Furthermore, the secondary service capacity in Modified-AMTPA is smaller than that of in AMTPA and it is even lower in AMTPA-CPE. For $M \geq 40$ the capacity of the secondary service in AMTPA-CPE approaches zero since the interference threshold $Q_{I}$ reduces to zero.

\section{Impact of Error in Primary Power Control Commands}

Here we also study the impact of error in power control commands. In Section IV-D we proposed a modification on AMTPA as Random-Modified-AMTPA. Fig. 12 shows the collision probability versus $p_{R M}$ for $M=30$ and various values of $p_{\text {error }}$, where $p_{\text {error }}$ is the probability of existence of error in the received power control command.

For $p_{\text {error }}=0$ the best collision performance is achieved when $p_{R M}=1$. This means that if the power control commands are errorless, the secondary service should adjust transmit power based on the captured commands. For $p_{\text {error }}=1$, the best collision performance is achieved for $p_{R M}=0$.

It is also interesting to examine the impact of the error in the primary service power control on the capacity of the secondary service. Fig. 13 shows the secondary service capacity versus 


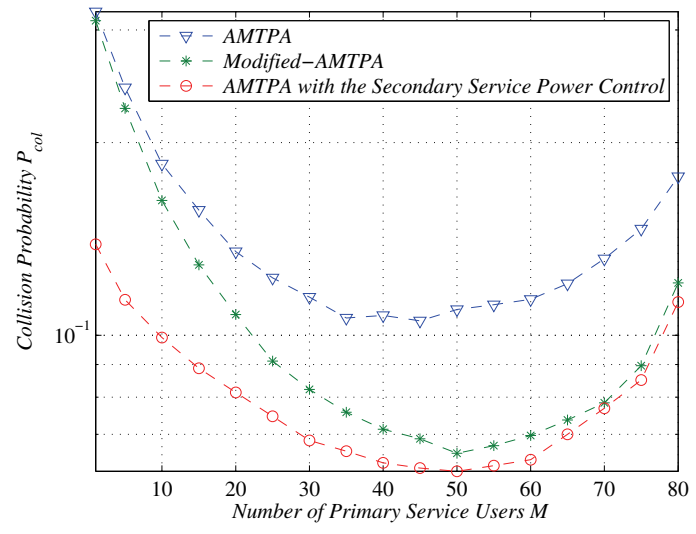

Fig. 14. Collision probability vs. $M$ for AMTPA, Modified-AMTPA and AMTPA with the secondary service power control.

$p_{R M}$ for $M=30$ and various values of $p_{\text {error. }}$. As it is also seen, in this case the trend of the capacity is similar to the observed collision probability behavior in Fig. 12.

\section{Impact of Secondary Service Power Control}

As mentioned before, the secondary service may also have a power control mechanism (see Section IV-E). Here we simulate a system in which the secondary service access point sends power control commands and the interference reduction power adjustment phase is based on (19). In Fig. 14 the collision probability is plotted versus $M$ for AMTPA, ModifiedAMTPA and AMTPA with secondary service power control. The required SINR at the secondary service receiver is 10 $\mathrm{dB}$. It is seen that by considering the secondary power control command the collision probability is reduced comparing to AMTPA and Modified-AMTPA.

In AMTPA, the secondary service is able to increase its transmission power in some circumstances, while it is not really required to do this based on the received secondary service power control commands. Therefore, as Fig. 14 also indicates the collision probability is decreased. It must be noted that, this behavior is related to the value of the required SINR at the secondary service receiver.

\section{E. Issuing Probability of an Increasing Power Control Com- mand in the Primary Service}

Issuing Probability of an Increasing Power Control Command (IPIPCC) in the primary system is related to the rate of violation of the minimum required SINR at the primary service. This violation may be caused by channel variations or co-channel interference. In this subsection we study the IPIPCC for different versions of AMTPA, system with no spectrum sharing, and a spectrum sharing system which only adopts rate optimal power allocation phase. The results have been plotted in Fig. 15.

The system with no spectrum sharing is assumed to be able to support $M_{0}$ users. For this system IPIPCC is upper bounded by 0.85 which is corresponding to fully loaded system with $M=M_{0}$. For the system which only adopts rate optimal power allocation phase Fig. 15 shows that the power control behavior is very similar to a fully loaded system even for very small values of $M$, i.e., $M \geq 5$. Consequently for a primary

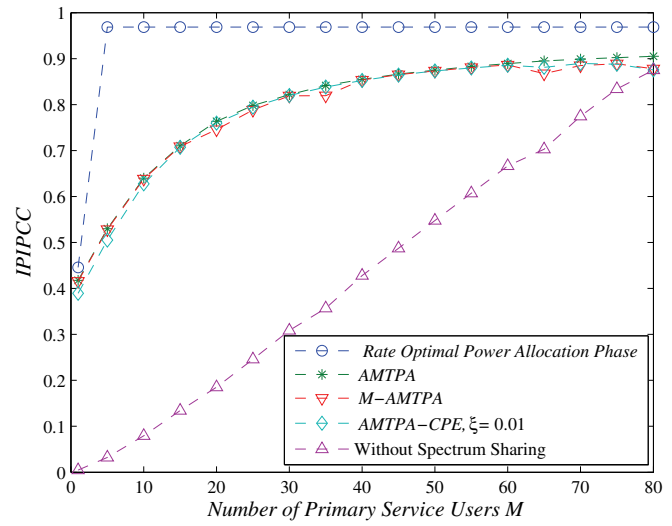

Fig. 15. IPIPCC vs. $M$ for system with no spectrum sharing, AMTPA without interference reduction power adjustment, system which also performs the interference reduction power adjustment phase (AMTPA and its modifications).

user in this system experiencing an outage has high probability. It is seen that conducting the interference reduction power adjustment (i.e., utilizing AMTPA) significantly changes the primary service power control behavior, so that the system experiences same power control behavior in very higher values of $M$ comparing to rate optimal power allocation system. This shows that using AMPTA the primary system with $M=40$ users acts similar to a system with no spectrum sharing with 80 users. Similar pattern is also observed for Modified-AMTPA and AMTPA-CPE. This shows that AMTPA and its variations enable spectrum sharing in a power controlled primary service.

Looking to the plot from another perspective, reveals the fact that using rate optimal power allocation spectrum sharing makes the primary system to act as an overloaded network from the power control viewpoint. However, AMTPA and its variations never degrades the IPIPCC performance lower than that of a fully loaded system with no spectrum sharing.

\section{CONCLUding REMARKS}

In this paper, the Adaptive Multiple Time-Scale Power Allocation (AMTPA) method for underlay spectrum sharing in DS-CDMA wireless networks was presented. In AMTPA, the secondary service has access to the power control commands transmitted by the BS of the primary service. AMTPA manages the secondary service transmission power in two phases, each in different time-scales: the rate optimal power allocation phase and the interference reduction power adjustment phase. In the longer time-scale, the rate optimal power allocation phase adaptively allocates the transmission power of the secondary service transmitter using the instantaneous status of the secondary channel, to maximize the achieved capacity. The allocated power in the rate optimal power allocation phase causes interference to the primary service. Then, in the shorter time-scale, the interference reduction power adjustment phase exploits the power control commands to adjust its transmission power with the objective of reducing the impact of the secondary service transmission on the qualityof-service in the primary network. Taking into account some practical considerations, we also proposed modified versions of AMTPA that have a smaller probability of collision and is more robust against errors in reception of the power control commands. 
Simulation results confirm our analysis and show that by using AMTPA, without using direct signaling between the two systems, spectrum sharing can be realized with a slight, but controllable level, of increase in the probability of collision. We also showed how one can set system parameters such as $\Delta_{s}$, and $p_{R M}$ so that the primary network collision probability constraint is satisfied.

\section{APPENDIX I: OBTAINING EQUATION (4)}

Consider the uplink of a DS-CDMA cellular network with $B \mathrm{~Hz}$ bandwidth. For mobile user $u_{j}$, the average $E_{b} / I_{0}$ is assumed to be $\rho_{j}$ which is

$$
\rho_{j}=\frac{B}{\nu_{j} R_{j}} \frac{Q_{j}}{Q_{\mathrm{total}}-Q_{j}},
$$

where $\nu_{j}, Q_{j}$, and $R_{j}$ are the activity factor, the average received power, and the bit rate of the $j$-th mobile user, respectively, and $Q_{\text {total }}$ is the total received power of users plus noise at the BS. The load factor of mobile user $j$ is denoted by $\eta_{j}$, where $Q_{j}=\eta_{j} Q_{\text {total }}$, and $\eta_{j}$ indicates the contribution of user $j$ in the total interference at the BS, $Q_{\text {total }}$. Using $\eta_{j}$ in (22), it is easy to verify

$$
\eta_{j}=\left(1+\frac{B}{\rho_{j} \nu_{j} R_{j}}\right)^{-1} .
$$

The total interference at the BS is divided into three different parts as $Q_{\text {total }}=Q_{\text {home }}+Q_{\text {other }}+N_{0} B$, where $Q_{\text {home }}$ is the interference generated by home cell users, $Q_{\text {other }}$ is the interference generated by the users in the other cells. In line with the DS-CDMA literature (see, e.g., [22]), we also assume that $Q_{\text {other }}=f Q_{\text {home }}$. The parameter $f$ defined as the ratio of other cell's interference to the home cell interference and called as network load factor. Consequently, we define $\eta_{\text {UP }}$ as the uplink load factor which shows the contribution of the home cell and other cell interference into $Q_{\text {total }}$ :

$$
\eta_{\mathrm{UP}}=\frac{Q_{\text {home }}+Q_{\text {other }}}{Q_{\text {total }}} .
$$

We note that $\eta_{\mathrm{UP}}=(1+f) \sum \eta_{j}$, then noting (23) for $S$ different services each with $n_{s}$ users, $\eta_{\mathrm{UP}}$ is obtained as

$$
\eta_{\mathrm{UP}}=(1+f) \sum_{s=1}^{S} n_{s} \eta_{s}=(1+f) \sum_{s=1}^{S} n_{s}\left(1+\frac{B}{\rho_{s} \nu_{s} R_{s}}\right)^{-1} .
$$

Rewriting $Q_{\text {total }}$ as $Q_{\text {total }}=\eta_{\mathrm{UP}} Q_{\text {total }}+N_{0} B$, and replacing $\eta_{\mathrm{UP}}$ from (25), $Q_{\text {total }}$ is obtained as

$$
Q_{\text {total }}=\left(1-(1+f) \sum_{s=1}^{S} n_{s}\left(1+\frac{B}{\rho_{s} \nu_{s} R_{s}}\right)^{-1}\right)^{-1} N_{0} B .
$$

Thus, parameter $K$ in $Q_{\text {total }}=K N_{0} B$, is obtained as it is presented in (4).

\section{APPENDIX II: Proof of Proposition 2}

Here we drop time index $n$ for brevity. Using (14) and (10), the collision probability is obtained as

$$
P_{c o l(i)}=\operatorname{Pr}\left\{g_{0 i} P_{s i, W F}>Q_{I}\right\},
$$

where $P_{s i, W F}$ is given by (12). Therefore,

$$
\begin{aligned}
P_{\text {col }(i)=} \int_{\boldsymbol{\Phi}} \operatorname{Pr}\left\{g_{0 i} P_{s i, W F}(\alpha)>Q_{I}, \alpha \geq \lambda_{0} \tilde{K} N_{0} B_{c}\right\} & \times f_{g_{1 i}}(\alpha) d \alpha \\
=\int_{\boldsymbol{\Phi}} \int_{\boldsymbol{\Psi}} f_{g_{1 i}}(\alpha) f_{g_{0 i}}(\beta) d \alpha d \beta, &
\end{aligned}
$$

where $\boldsymbol{\Psi}=\left\{\beta \geq \frac{Q_{I}}{\frac{1}{\lambda_{0}}-\frac{K N_{0} B_{c}}{\alpha}}\right\}$. In the above, $g_{0 i}$ and $g_{1 i}, i=1, \ldots, N$, are i.i.d. random variables with $\log$ normal distribution. For a log-normal random variable $X$ with variance $\sigma_{d B}, X=e^{Z}$, where $Z$ is a zero-mean Gaussian random variable with variance $\sigma^{2}$, and $\sigma=0.1 \log _{10} \sigma_{d B}$; therefore,

$$
f_{X}(x)=\frac{1}{x \sigma \sqrt{2 \pi}} \exp \left(-\frac{(\ln x)^{2}}{2 \sigma^{2}}\right) .
$$

Substituting pdf of $g_{0 i}$, i.e., (30), into (29) yields (15) and completes the proof.

\section{REFERENCES}

[1] J. M. Peha, "Approaches to spectrum sharing," IEEE Commun. Mag., pp. 10-12, Feb. 2005.

[2] Q. Zhao and B. Sadler, "A survey of dynamic spectrum access: signal processing, networking, and regulatory policy," IEEE Signal Process. Mag., vol. 24, no. 3, pp. 79-89, May 2007.

[3] M. G. Khoshkholgh, K. Navaie, and H. Yanikomeroglu, "Access strategies for spectrum sharing in fading environment: overlay, underlay and mixed," IEEE Trans. Mobile Comput., vol. 9, no. 12, pp. 1780-1793, Dec. 2010.

[4] S. Srinivasa and S. A. Jafar, "Soft sensing and optimal power control for cognitive radio," in Proc. 2007 IEEE Global Communications Conference, pp. 26-30.

[5] M. G. Khoshkholgh, K. Navaie, and H. Yanikomeroglu, "On the impact of the primary network activity on the achievable capacity of spectrum sharing over fading channels," IEEE Trans. Wireless Commun., vol. 8, no. 4, pp. 2100-2111, Apr. 2009.

[6] - "Impact of the primary network activity on the maximum achievable capacity of DS-CDMA/OFDM spectrum sharing," in Proc. 2008 IEEE Vehicular Technology Conference - Fall.

[7] R. Etkin, A. Parekh, and D. Tse, "Spectrum sharing for unlicensed bands," IEEE J. Sel. Areas Commun., vol. 25, no. 3, pp. 517-528, Apr. 2007.

[8] L. Zhang, Y.-C. Liang, and Y. Xin, "Joint beamforming and power allocation for multiple access channels in cognitive radio networks," IEEE J. Sel. Areas Commun., vol. 26, no. 1, pp. 38-51, Jan. 2008.

[9] P. Stocia and T. Sundia, "On non-parametric spectral estimation," Circuits, Systems and Signal Process., vol. 16, pp. 169-181, 1999.

[10] S. Haykin, "Cognitive radio: brain-empowered wireless communications," IEEE J. Sel. Areas Commun., vol. 23, no. 2, pp. 201-220, Feb. 2005.

[11] K. Eswaran, M. Gastpar, and K. Ramchandran, "Cognitive radio through primary control feedback," IEEE J. Sel. Areas Commun., vol. 29, no. 2, pp. 384-393, 2011.

[12] K. G. Seddik, A. K. Sultan, A. A. El-Sherif, and A. M. Arafa, "Feedback-based access scheme for cognitive radio systems," in Proc. 2011 IEEE International Workshop on Signal Processing Advances in Wireless Communications.

[13] J. Li, W. Zhang, and J. Yuan, "Opportunistic spectrum sharing in cognitive radio networks based on primary limited feedback," IEEE Trans. Commun., vol. 12, no. 59, pp. 3272-3277, 2011.

[14] S. Huang, X. Liu, and Z. Ding, "Decentralized cognitive radio control based on inference from primary link control information," IEEE J. Sel. Areas Commun., vol. 29, no. 2, pp. 394-406, 2011.

[15] D. Tse and P. Viswanath, Fundamentals of Wireless Communication. Cambridge University Press, 2004.

[16] A. J. Viterbi, CDMA: Principles of Spread Spectrum Communication. Addison-Wesley, 1995.

[17] R. Steele, C. C. Lee, and P. Gould, GSM, cdmaOne and $3 G$ Systems. John Wiley and Sons, 2001. 
[18] S. Ariyavisitakul, "SIR based power control in a CDMA system," in Proc. 1992 IEEE GLOBECOM, pp. 868-873.

[19] A. Papoulis and S. U. Pillai, Probability, Random Variables, and Stochastic Processes, 4th ed. McGraw-Hill, 2002.

[20] Z. Quan, S. Cui, and A. H. Sayed, "Optimal linear cooperation for spectrum sensing in cognitive radio networks," IEEE J. Sel. Topics Signal Process., vol. 2, no. 1, pp. 28-40, Feb. 2008.

[21] H. Holma and A. Toskala, WCDMA for UMTS: Radio Access for Third Generation Mobile Communications. John Willy and Sons, 2000.

[22] K. Navaie and A. R. Sharafat, "A framework for UMTS air interface analysis," Canadian J. Electrical and Computer Engineering, vol. 28 , no. 2, pp. 113-129, July/Oct. 2003.

[23] K. Navaie, H. Yanikomeroglu, M. G. Khoshkholgh, A. R. Sharafat, and H. Nikoofar, Cognitive Radio Mobile Ad Hoc Networks. Springer, 2011, ch. DS-CDMA/OFDM spectrum sharing.

[24] A. J. Goldsmith and P. P. Varaiya, "Capacity of fading channels with channel side information," IEEE Trans. Inf. Theory, vol. 43, no. 6, pp. 1986-1992, Nov. 1997.

[25] M. G. Khoshkholgh, K. Navaie, and H. Yanikomeroglu, "Achievable capacity in DS-CDMA OFDM spectrum-sharing," IEEE Trans. Mobile Comput., vol. 9, no. 6, pp. 765-777, 2010.

[26] M. Rintamki, "Adaptive power control in CDMA cellular communication systems," Ph.D. dissertation, Helsinki University of Technology, Espoo, Finland, 2004.

[27] J. Zander, "Performance of optimum transmitter power control in cellular radio systems," IEEE Trans. Veh. Technol., vol. 41, no. 1, pp. 57-62, Feb. 1992.

[28] T. H. Lee, J. C. Lin, and Y. T. Su, "Downlink power control algorithms for cellular radio systems," IEEE Trans. Veh. Technol., vol. 44, no. 1, pp. 89-94, Feb. 1995

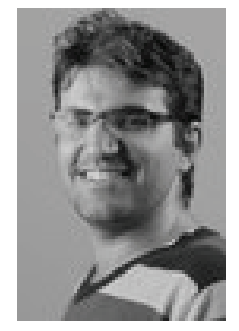

Mohammad G. Khoshkholgh received his B.Sc. degree in Electerical Engineering from Isfahan University, Isfahan, Iran, in 2006, his M.Sc. degree in Electrical Engineering from Tarbiat Modares University, Tehran, Iran, in 2008. From 2008 to 2012 he was with Wireless Innovation Laboratory (WIL) in Tarbiat Modares University. He is currently with Simula Research Laboratory, Fornebu, Norway. His research interests are mainly in wireless communications, radio resource allocations, and spectrum sharing.

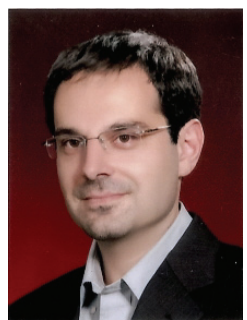

Keivan Navaie (S'02-M'05-SM'10) received his B.Sc. degree from Sharif University of Technology, Tehran, Iran, his M.Sc. degree from the University of Tehran, Tehran, Iran, and his Ph.D. degree from Tarbiat Modares University, Tehran, Iran, all in Electrical Engineering in 1995, 1997 and 2004 respectively. From March to November 2004, he was with the School of Mathematics and Statistics, Carleton University, Ottawa, Canada, as a Postdoctoral Research Fellow. From December 2004 to September 2006, he was with the Broadband Communication and Wireless System (BCWS) Centre, Carleton University, Ottawa, Canada where he was the project manager of BCWS participation in European Union 6th Framework integrated project, the Wireless World Initiative New Radio (WINNER) on beyond 3G wireless systems. From September 2006 to July 2011 he was with the Department of Electrical and Computer Engineering, Tarbiat Modares University, Tehran, Iran. Since July 2011 he has been with the School of Electrical and Computer Engineering, University of Leeds, Leeds, UK.
His research interests lie in the field of radio resource allocation for wireless communication systems, dynamic spectrum allocation, cognitive radio networks and cooperative communications. Dr. Navaie is on the editorial board of the European Transactions on Telecommunications. He has been on the technical program committee of different IEEE conferences, including Globecom, ICC, VTC and WCNC, and chaired some of their symposia. $\mathrm{He}$ has also served as Co-Chair of Wireless Network Track, IEEE VTC2012 Yokohama, Japan and IEEE 8th International Workshop on Wireless Network Measurements WiNMee 2012, Paderborn, Germany, IEEE. He is also Co-Chair of Wireless Networks and Security Track, IEEE VTC2014-Spring, Seoul, South Korea and Co-Chair of the Mobile and Wireless Networks Track, IEEE WCNC 2014, Istanbul, Turkey. He is the recipient of the 2011 IEEE Iran Section Young Investigator award. Dr. Navaie is a senior member of the IEEE.

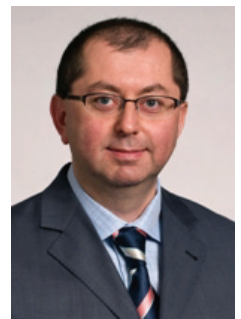

Halim Yanikomeroglu (S'96-M'98-SM'12) was born in Giresun, Turkey, in 1968. He received the B.Sc. degree in Electrical and Electronics Engineering from the Middle East Technical University, Ankara, Turkey, in 1990, and the M.A.Sc. degree in Electrical Engineering (now ECE) and the Ph.D. degree in Electrical and Computer Engineering from the University of Toronto, Canada, in 1992 and 1998, respectively. He was with the R\&D Group of Marconi Kominikasyon A.S., Ankara, Turkey, during 1993-1994.

Since 1998 Dr. Yanikomeroglu has been with the Department of Systems and Computer Engineering at Carleton University, Ottawa, where he is now a Full Professor. His research interests cover many aspects of wireless technologies with a special emphasis on cellular networks. Dr. Yanikomeroglu coauthored more than 50 journal and 160 conference papers, and has given more than 20 tutorials in leading international conferences on wireless technologies. In recent years, Dr. Yanikomeroglus research has been funded by Huawei, BlackBerry (formerly Research in Motion (RIM)), Samsung, Communications Research Centre of Canada (CRC), Telus, and Nortel. This collaborative research resulted in about 15 patent applications.

Dr. Yanikomeroglu is a member of the Steering Committee of the IEEE Wireless Communications and Networking Conference (WCNC), and has been involved in the organization of this conference over the years, including serving as the Technical Program Co-Chair of WCNC 2004 and the Technical Program Chair of WCNC 2008; he will also serve as the Technical Program Co-Chair of WCNC 2014 to be held in Istanbul. Dr. Yanikomeroglu was the General Co-Chair of the IEEE Vehicular Technology Conference Fall 2010 held in Ottawa.

Dr. Yanikomeroglu has served in the editorial boards of the IEEE TRANSACTIONS ON COMMUNICATIONS, IEEE TRANSACTIONS ON WIRELESS Communications, and IEEE Communications Surveys \& TUTORIALS. He was the Chair of the IEEE Technical Committee on Personal Communications (now called, Wireless Technical Committee). He is a Distinguished Lecturer for the IEEE Vehicular Technology Society. Dr. Yanikomeroglu is a recipient of the Carleton University Faculty Graduate Mentoring Award in 2010, the Carleton University Graduate Students Association Excellence Award in Graduate Teaching in 2010, and the Carleton University Research Achievement Award in 2009. Dr. Yanikomeroglu spent the 2011-2012 academic year at TOBB University of Economics and Technology, Ankara, Turkey, as a Visiting Professor. He is a registered Professional Engineer in the province of Ontario, Canada. 